\title{
Cell division cycle-associated protein 1 overexpression is essential for the malignant potential of colorectal cancers
}

\author{
YU KOBAYASHI ${ }^{1,3}$, ATSUSHI TAKANO ${ }^{1,2}$, YOHEI MIYAGI ${ }^{6}$, EIJU TSUCHIYA ${ }^{6}$, HIROMICHI SONODA ${ }^{4}$, \\ TOMOHARU SHIMIZU ${ }^{4}$, HIDETOSHI OKABE ${ }^{5}$, TORU TANI ${ }^{4}$, YOSHIHIDE FUJIYAMA ${ }^{3}$ and YATARO DAIGO ${ }^{1,2}$ \\ ${ }^{1}$ Department of Medical Oncology and ${ }^{2}$ Cancer Center, Departments of ${ }^{3}$ Medicine, ${ }^{4}$ Surgery and \\ ${ }^{5}$ Clinical Laboratory Medicine, Shiga University of Medical Science, Otsu; \\ ${ }^{6}$ Kanagawa Cancer Center, Research Institute, Yokohama, Japan
}

Received September 4, 2013; Accepted October 21, 2013

DOI: $10.3892 /$ ijo.2013.2177

\begin{abstract}
To identify new cancer biomarkers and therapeutic targets for colorectal cancers (CRCs), we performed immunohistochemical analysis using tissue microarrays covering archival tumor tissue samples from 434 CRC patients and antibodies to cell division cycle-associated protein 1 (CDCA1) that was originally identified as an oncoantigen by our gene expression profile database, and compared its expression with several clinicopathological factors. Strong CDCA1 positivity was associated with poorer prognosis for patients with CRC $(\mathrm{P}=0.019)$ and multivariate analysis confirmed its independent prognostic value. In addition, transfection of siRNAs against CDCA1 suppressed its expression and induced apoptosis of CRC cells. These results suggest that CDCA1 could be a prognostic biomarker and a potential therapeutic target for CRCs.
\end{abstract}

\section{Introduction}

Colorectal cancer (CRC) is the third most commonly diagnosed cancer in males and the second in females worldwide, and is the fourth leading cause of cancer death in males and the third in females worldwide (1). Although several molecular target therapies have been developed for CRCs, the prognosis of this disease in advanced stages is still poor. Therefore, personalized therapies using biomarkers and new molecular therapies are expected to improve current therapies. For example, cetuximab, a therapeutic antibody to an epidermal growth factor receptor (EGFR), was reported to be effective in CRC treatment. The randomized phase III study (CRYSTAL: Cetuximab Combined with Irinotecan in First-Line Therapy for Metastatic Colorectal Cancer) and randomized phase II study (OPUS: Oxaliplatin and Cetuximab in First-Line Treatment of metastatic CRC) demonstrated that addition of cetuximab to first-line chemotherapy

Correspondence to: Dr Yataro Daigo, Department of Medical Oncology, Shiga University of Medical Science, Seta Tsukinowa-cho, Otsu 520-2192, Japan

E-mail: ydaigo@belle.shiga-med.ac.jp

Key words: cell division cycle-associated protein 1, oncoantigen, molecular target, biomarker, colorectal cancer in patients with KRAS wild-type CRC significantly improved the treatment outcome compared with chemotherapy alone. However, the benefit of cetuximab was limited to patients with KRAS wild-type tumors. The overall survival (OS) rate in these trials was still less than two years, and adverse events such as skin reactions, infusion-related reactions and diarrhea were more frequent with cetuximab plus chemotherapy than with chemotherapy alone $(2,3)$. Therefore, there is a need for personalized therapies that are more effective for CRCs including the KRAS mutant, but with fewer adverse events.

Oncoantigens are proteins with oncogenic functions that are overexpressed in malignant cells of various origins and in normal reproductive tissues such as the testis, ovary, and placenta $(4,5)$. Oncoantigens are considered a candidate biomarker and therapeutic target for cancer therapy. We performed genome-wide gene expression analyses and subsequent tissue microarray analyses of solid tumor tissues using a cDNA microarray containing 25,000 genes or expressed sequence tags (ESTs) (6-10). To date, we have isolated several oncoantigens involved in development and/or progression of cancer (11-42). These data revealed that cell division cycleassociated protein $1(C D C A 1)$ was overexpressed in cancer tissues including $\mathrm{CRC}$ and lung cancer tissues. In our previous reports, CDCA1 proteins were detected in many lung cancers with varying histologic types and were associated with a poorer prognosis for patients with non-small cell lung carcinomas (NSCLC). Knockdown of CDCA1 expression with siRNA significantly suppressed growth of NSCLC cell lines (16). In addition, the HLA-A0201-restricted peptides derived from CDCA1 induced peptide-specific cytotoxic T lymphocytes (CTLs), suggesting that CDCA1 is a likely target for molecular therapy and/or immunotherapy (39). CDCAI mRNA expression was also observed in CRCs and tissues of gastric cancers, and was correlated with cancer growth (43). Moreover, CDCA1 was associated with a decrease in progression-free survival of multiple myeloma patients, and a decrease in probability of biochemical-free survival in localized prostate cancer (44).

CDCA1 plays a role in regulating mitosis. CDCA1, and its binding partner KNTC2, are members of the Ndc80 complex, which comprises the two Ndc80 sub-complexes (KNTC2)-Nuf2 (CDCA1) and Spc24-Spc25 (46). The CDCA1-KNTC2 
complex is highly conserved in prokaryotic and eukaryotic cells, and plays an important role in kinetochore functions and the spindle checkpoint (46). Although some reports describe $C D C A 1$ expression in human cancers, no report has revealed the function of CDCA1 in colon cancer growth/survival in detail, or the clinical or prognostic value of CDCA1 protein as a tissue biomarker for various colon cancers.

We present evidence that CDCA1 plays a significant role in the malignant potential of $\mathrm{CRC}$, and is a promising diagnostic and prognostic biomarker, as well as a therapeutic target for treating CRC.

\section{Materials and methods}

Colorectal cancer cell lines and tissue samples. The human CRC cell lines, Caco-2, CCD-841, COLO205, LoVo, HCT116, HT-29, SW48, SW480, SW620 and SW948 were purchased from American Type Culture Collection (ATCC; Rockville, MD, USA). All cells were grown in monolayers in the appropriate medium (Table I), supplemented with $10 \%$ fetal bovine serum (FBS) (Nichirei Biosciences, Tokyo, Japan), and were maintained at $37^{\circ} \mathrm{C}$ in atmospheres of humidified air. Eight CRC tissue samples and adjacent normal colorectal tissue samples were obtained from patients undergoing surgery at Shiga University of Medical Science Hospital. In addition, we obtained $434 \mathrm{CRC}$ and adjacent normal colorectal tissue samples for immunostaining on tissue microarrays from CRC patients without distant metastases who underwent surgery at Kanagawa Cancer Center Hospital. Individual institutional ethics committees approved this study and the use of all clinical materials.

Semi-quantitative reverse transcription-PCR. Total RNAs were extracted from cultured cells and clinical tissues using Maxwell 16 LEV simplyRNA Purification kit (Promega, Madison, WI, USA) according to the manufacturer's protocol. The RNAs from cultured cells and clinical tissues, as well as commercially available mRNAs from normal human colon and rectum samples (Agilent Technologies, Santa Clara, CA, USA) were reversely transcribed using ReverTra Ace ${ }^{\circledR}$ qPCR RT kit (Toyobo, Osaka, Japan). Semi-quantitative reverse transcription-PCR (RT-PCR) experiments were carried out with the following synthesized $C D C A 1$-specific primers or with $\beta$-actin (ACTB)-specific primers as an internal control: CDCA1, 5'-GAGAAACTGAAGTCCCAGGAAAT-3' and 5'-CTGATACTTCCATTCGCTTCAAC-3'; ACTB, 5'-GCC ACCCCACTTCTCTCTAA-3' and 5'-CACGAAGGCTCAT CATTCAA-3'. RT-PCR reactions were optimized for the number of cycles to ensure product intensity within the logarithmic phase of amplification.

Western blot analysis. Cells were lysed in Pierce RIPA buffer (Thermo Scientific, Waltham, MA, USA) that included $1 \%$ protease inhibitor cocktail (Thermo Scientific). After homogenization, the cell lysates were incubated on ice for $30 \mathrm{~min}$ and centrifuged at $14,000 \mathrm{rpm}$ for $15 \mathrm{~min}$ to separate the supernatant from cellular debris. The amount of total protein was estimated using a Quick Start Bradford Protein Assay kit (Bio-Rad, Hercules, CA, USA), and the proteins were then mixed with SDS sample buffer and incubated at
Table I. The human CRC cell lines.

\begin{tabular}{ll}
\hline Cell line & \multicolumn{1}{c}{ Medium } \\
\hline Caco-2 & Eagle's minimum essential medium \\
CCD-841 & Dulbecco's modified Eagle's medium \\
COLO205 & RPMI-1640 medium \\
LoVo & F-12K medium \\
HCT116 & McCoy's 5a medium modified \\
HT-29 & McCoy's 5a medium modified \\
SW48 & Leibovitz's L-15 medium \\
SW480 & Leibovitz's L-15 medium \\
SW620 & Leibovitz's L-15 medium \\
SW948 & Leibovitz's L-15 medium
\end{tabular}

All cells were purchased from American Type Culture Collection (ATCC; Rockville, MD, USA).

$37^{\circ} \mathrm{C}$ for $15 \mathrm{~min}$ before loading them into a $12 \%$ SDS-PAGE gel. After electrophoresis, the proteins were transferred onto an Amersham Hybond-P PVDF Membrane (GE Healthcare, Buckinghamshire, UK). Membranes were blocked using 4\% Block Ace (Dainippon Pharmaceutical, Osaka, Japan), and incubated with anti-Nuf2 (alias CDCA1) antibody (catalog no. ab96147; Abcam, Cambridge, MA, USA) and mouse anti- $\beta$-actin antibody (catalog no. 8H10D10; Cell Signaling Technology, Danvers, MA, USA). In the final step, the membranes were incubated with enhanced chemiluminescence (ECL) anti-rabbit IgG, horseradish peroxidase (HRP)-linked whole antibody, ECL anti-mouse IgG and HRP-linked whole antibody (GE Healthcare). Protein bands were visualized using ECL detection reagents (GE Healthcare).

Immunocytochemistry. Cultured cells were washed twice with PBS(-), fixed in $4 \%$ formaldehyde solution for $30 \mathrm{~min}$ at room temperature and rendered permeable by a 3-min treatment with PBS(-) containing 0.1\% Triton X-100. Cells were covered with CAS Block (Invitrogen, Carlsbad, CA, USA) for 7 min to block non-specific binding before the primary antibody reaction. Then the cells were incubated with anti-Nuf 2 antibody. The immune complexes were stained with Alexa Fluor 488 goat anti-rabbit IgG (Life Technologies, Grand Island, NY, USA) and mounted with Vectashield mounting medium with DAPI (Vector Laboratories, Burlingame, CA, USA), and viewed using a motorized inverted microscope IX81 (Olympus, Tokyo, Japan).

Immunohistochemistry and tissue microarray analysis. To investigate the significance of CDCA1 overexpression in clinical CRCs, we stained tissue sections using EnVision ${ }^{+}$kit/ horseradish peroxidase (HRP; DakoCytomation, Glostrup, Denmark). Anti-Nuf2 antibody was added after blocking of endogenous peroxidase and proteins and each section was incubated with HRP-labeled anti-rabbit IgG as the secondary antibody. Substrate-chromogen was added and the specimens were counterstained with hematoxylin and eosin (HE). Tumor tissue microarrays were constructed according to previously published procedures, using formalin-fixed CRCs that were surgically resected (11-13). Tissue areas selected for sampling 
A

Clinical CRC tissues

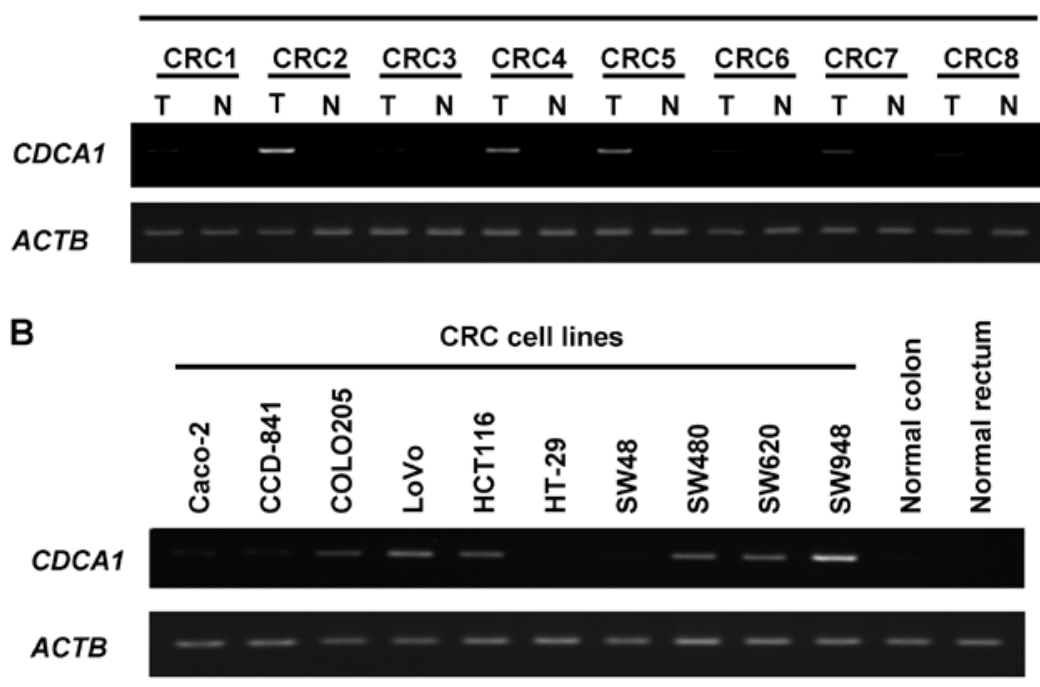

Figure 1. CDCA1 gene expression in human CRC cell lines and tissues. (A) CDCA1 mRNA expression in tumor tissues (T) and adjacent normal colorectal tissues (N) from eight CRC patients (cases 1-8) examined using semi-quantitative RT-PCR. ACTB expression served as a quantity control. (B) CDCA1 mRNA expression in $10 \mathrm{CRC}$ cell lines and normal human colorectal tissues examined using semi-quantitative RT-PCR.

were determined by the visual alignment with the corresponding HE-stained sections on slides. Three, four or five tissue cores (diameter, $0.6 \mathrm{~mm}$; height, 3-4 mm) taken from donor tumor blocks were placed into recipient paraffin blocks using a tissue microarrayer (Beecher Instruments, Sun Prairie, WI, USA). A core of normal tissue was punched from each case. Sections (5- $\mu \mathrm{m}$ thick) of the resulting microarray block were used for immunohistochemical analysis. Positivity and staining intensity were recorded as absent, weak or strongly positive.

Statistical analysis. We used contingency tables to correlate clinicopathological variables, such as gender, age, histologic type, and pathologic tumor-node-metastasis (TNM) stage, with CDCA1 protein expression levels determined by tissue microarray analysis. Survival curves were calculated from the surgery date to the CRC-related time of death or to the last follow-up observation. Kaplan-Meier curves were calculated for each relevant variable and for CDCA1 expression; differences in survival times among patient subgroups were analyzed using the log-rank test. Univariate analysis was performed using the Cox proportional-hazard regression model to determine associations between clinicopathological variables and mortality. We first analyzed associations between death and possible prognostic factors including age, gender, pathologic tumor classification and pathologic node classification, taking into consideration one factor at a time. Then, a multivariate Cox analysis was applied on backward (stepwise) procedures that always forced strong CDCA1 expression into the model, along with any or all variables that satisfied an entry level P-value of $<0.05$. As the model continued to add factors, independent factors did not exceed an exit level of $\mathrm{P}<0.05$.

Effect of small-interfering RNA against CDCA1 on CRC cell growth. To evaluate the biological functions of CDCA1 in CRC cells, we used siRNA oligonucleotides (Sigma-Aldrich Japan, Tokyo, Japan). The sequences targeting each gene were as follows: si-CDCA1-1, 5'-AAUGCCAGACAAGAAGUG GUG-3'; si-CDCA1-2, 5'-AAGAUGCUGCUGAAAGGG AGA-3'; si-EGFP, 5'-GAAGCAGCACGACUUCUUC-3'; and si-LUC, 5'-CGUACGCGGAAUACUUCGA-3'. CRC cells, SW480 or SW948, were transfected with either of siRNAs (si-CDCA1-1 or siCDCA1-2) using Lipofectamine 2000 reagent (Invitrogen) according to the manufacturer's instructions. Cell viability was measured using triplicate 3-(4,5-dimethylthiazol2-yl)-2,5-diphenyltetrazolium bromide (MTT) assays (Cell Counting kit-8 solution; Dojindo Laboratories, Kumamoto, Japan) after the transfection. Flow cytometric analysis was performed using CycleTest Plus DNA reagent kit and FACSVerse system (BD Immunocytometry Systems, San Jose, CA, USA). Caspase 3/7 assay (Caspase-Glo 3/7 assay; Promega) and TUNEL assay (DeadEnd Fluorometric TUNEL System; Promega) were performed according to the manufacturer's instructions. Endogenous CDCA1 protein expression was detected using western blotting.

\section{Results}

CDCAl gene expression in CRC tissues and cell lines. We previously demonstrated using gene expression profile analysis that $C D C A 1$ was overexpressed in cancer tissues including CRC tissues (data not shown). We also checked Gene Expression Omnibus (GEO; http://www.ncbi.nlm.nih.gov/ geo/; ID: 88360759) and found that $C D C A 1$ gene expression in colorectal cancer tissues was higher in all of 17 clinical CRC tissues than in their corresponding normal tissues. Because our original gene expression profile database and the publicly available database revealed high $C D C A 1$ expression levels in clinical CRCs, we performed a semi-quantitative RT-PCR analysis of $C D C A 1$ gene expression in both the cancer and corresponding normal colorectal tissues isolated from $8 \mathrm{CRC}$ patients and the $10 \mathrm{CRC}$ cell lines. CDCA1 gene was highly expressed in 4 of the $8 \mathrm{CRC}$ tissues and in 6 of the $10 \mathrm{CRC}$ cell lines, but was not detected in any normal colorectal tissues (Fig. 1). 
A

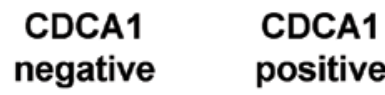

HT-29 SW480 SW620 SW948 kDa

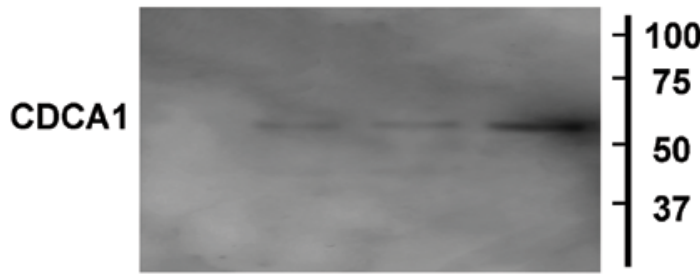

ACTB

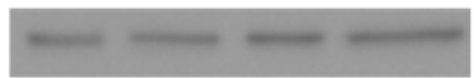

\section{B CDCA1 negative}
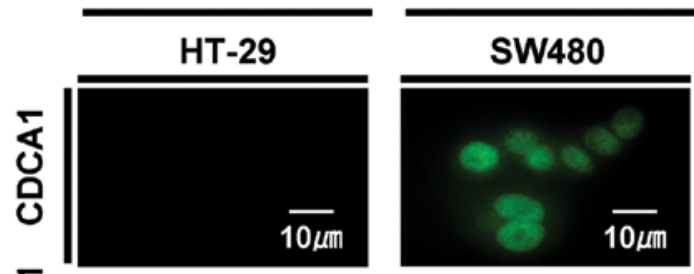

CDCA1 positive
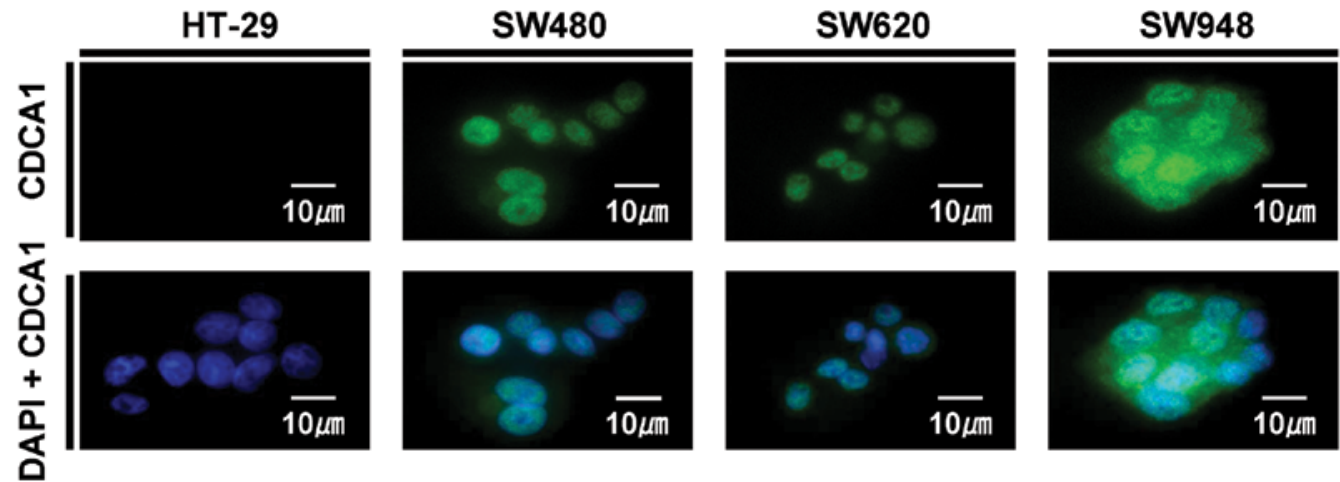

Figure 2. CDCA1 protein expression in CRC cell lines. (A) CDCA1 protein expression in four CRC cell lines examined using western blot analysis. ACTB expression served as a quantity control. (B) Subcellular localization of CDCA1 protein. The cells were immunocytochemically stained using anti-CDCA1 antibody (green) and DAPI (blue).

A

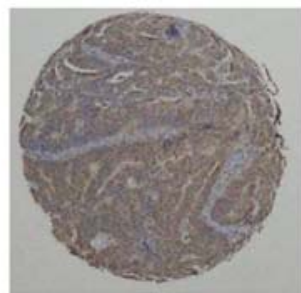

Strong expression
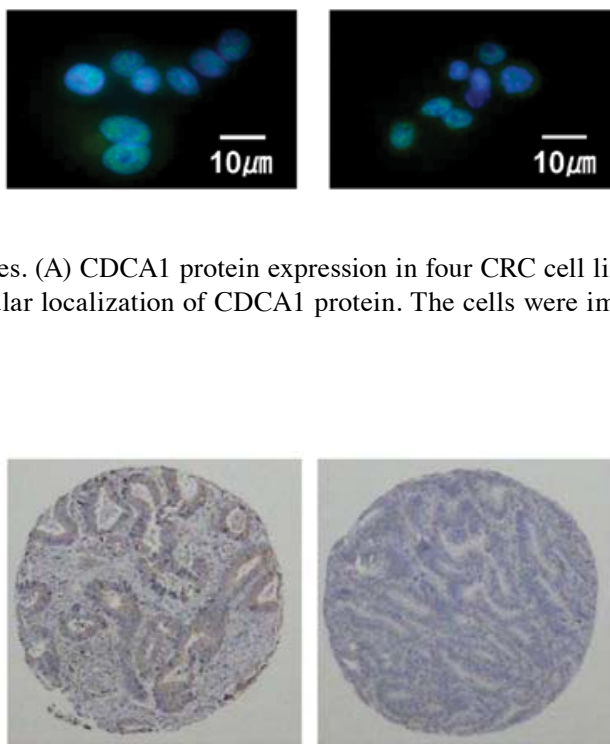

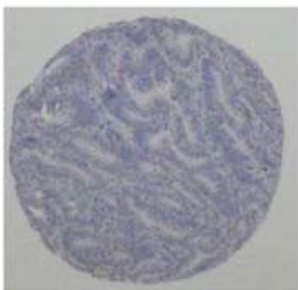

Weak expression Absent expression

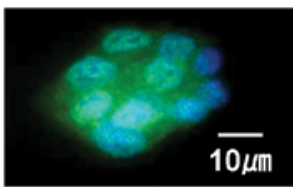

B

อ

Number at risk

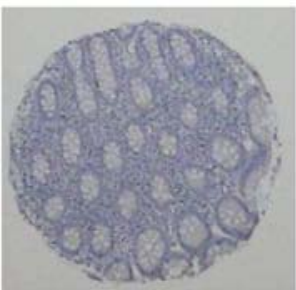

Normal

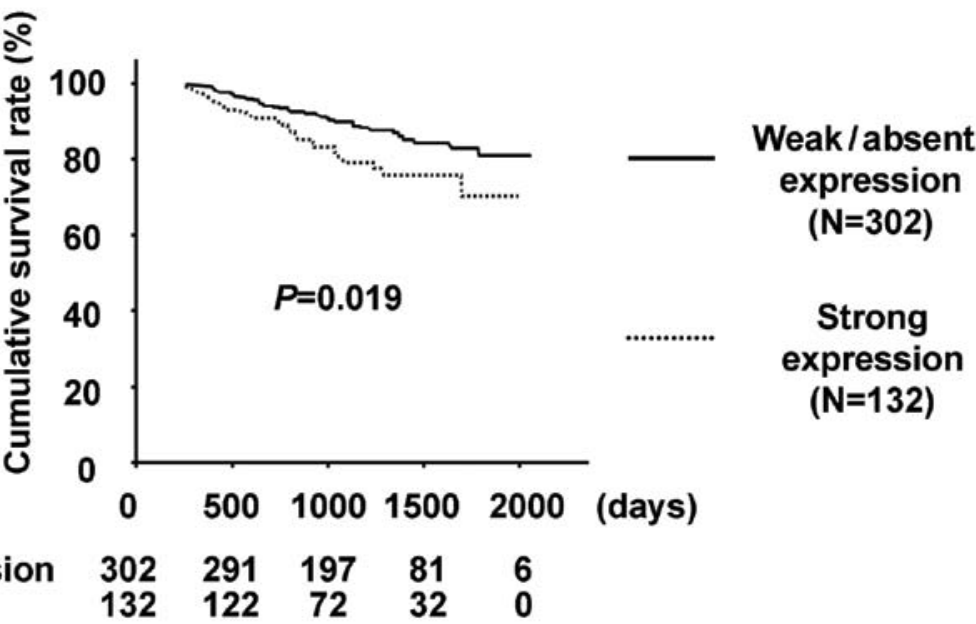

Figure 3. CDCA1 protein expression in CRC tissues and its association with poor clinical outcomes for CRC patients. (A) Immunohistochemical evaluation of CDCA1 expression on tumor tissue microarrays. Examples are strong, weak and absent CDCA1 expression in cancer tissues, and no expression in normal tissues. (Original magnification, x100). (B) Kaplan-Meier analysis of survival of patients with CRC (P=0.019 by the log-rank test) according to the CDCA1 expression levels. 
Table II. Association between CDCA1 positivity in colorectal cancer tissues and patient characteristics $(n=434)$.

\begin{tabular}{|c|c|c|c|c|c|}
\hline & $\mathrm{n}=434$ & $\begin{array}{c}\text { CDCA1 } \\
\text { Strong positive } \\
n=132\end{array}$ & $\begin{array}{c}\text { CDCA1 } \\
\text { Weak positive } \\
n=108\end{array}$ & $\begin{array}{c}\text { CDCA1 } \\
\text { Absent } \\
n=194\end{array}$ & $\begin{array}{c}\text { P-value } \\
\text { (Strong versus } \\
\text { weak, absent) }\end{array}$ \\
\hline \multicolumn{6}{|l|}{ Gender } \\
\hline Male & 249 & 74 & 73 & 102 & 0.752 \\
\hline Female & 185 & 58 & 35 & 92 & \\
\hline \multicolumn{6}{|l|}{ Age (years) } \\
\hline$<65$ & 215 & 74 & 54 & 87 & 0.077 \\
\hline$\geq 65$ & 219 & 58 & 54 & 107 & \\
\hline \multicolumn{6}{|l|}{ Histologic type } \\
\hline tub1, tub2, pap & 385 & 116 & 99 & 170 & 0.743 \\
\hline por1, por2, sig, others & 49 & 16 & 9 & 24 & \\
\hline \multicolumn{6}{|l|}{ pT factor } \\
\hline Tis+T1+T2 & 132 & 43 & 32 & 57 & 0.571 \\
\hline $\mathrm{T} 3+\mathrm{T} 4$ & 302 & 89 & 76 & 137 & \\
\hline \multicolumn{6}{|l|}{$\mathrm{pN}$ factor } \\
\hline $\mathrm{N} 0+\mathrm{N} 1$ & 364 & 108 & 89 & 167 & 0.479 \\
\hline $\mathrm{N} 2$ & 70 & 24 & 19 & 27 & \\
\hline
\end{tabular}

tub, tubular adenocarcinoma; tub1, well-differentiated type; tub2, moderately-differentiated type; pap, papillary adenocarcinoma; por, poorlydifferentiated adenocarcinoma; por1, solid type; por2, non-solid type; sig, signet ring cell carcinoma.

CDCA1 protein expression and its subcellular localization in CRC cells. To determine the CDCA1 protein expression and its subcellular localization in CRC cells, we performed western blotting and immunofluorescence analyses using antiCDCA1 antibody, CDCA1-positive CRC cells (SW480, SW620 and SW948), and CDCA1-negative HT-29 cells. The band was detected using western blotting in CDCA1-positive SW480, SW620 and SW948 cells, whereas no signal was detected in CDCA1-negative HT-29 cells (Fig. 2A). In addition, through an immunofluorescence analysis, we detected CDCA1 protein primarily in the nucleus and cytoplasm of CDCA1-positive SW480, SW620 and SW948 cells, but not in CDCA1-negative HT-29 cells (Fig. 2B).

Association of CDCA1 overexpression with poor clinical outcomes for CRC patients. To verify the biological and clinicopathological significance of CDCA1 in clinical CRCs, we examined CDCA1 protein expression with immunohistochemical analysis using anti-CDCA1 antibody and tissue microarrays for the $434 \mathrm{CRC}$ cases without distant metastases that underwent surgical resection. CDCA1 staining was observed primarily in the cell nucleus and cytoplasm of tumor cells, but was hardly detectable in surrounding normal tissues (Fig. 3A). We classified a CDCA1 expression pattern in the tissue array ranging from absent/weak to strong. Positive staining was observed in 240 of the 434 (55.3\%) CRC cases, whereas no staining was observed in adjacent normal tissues. Of the 434 CRC cases examined, CDCA1 was strongly stained in $132(30.4 \%)$, weakly stained in 108 (24.9\%) and unstained in 194 (44.7\%; Table II). CRC patients whose tumors showed strong CDCA1 expression had shorter survival compared with those with weak/absent CDCA1 expression $(\mathrm{P}=0.019$ by the log-rank test; Fig. 3B). We also applied univariate analysis to evaluate associations between patient prognosis and other factors, including gender (male versus female), age ( $<65$ years versus $\geq 65$ years), histologic type (tub1, tub2, pap versus por1, por2, sig, others), pT factor (Tis, T1, T2 versus T3, T4), pN factor (N0, N1 versus N2), and CDCA1 status (weak, absent versus strong). Among these variables, CDCA1 status $(\mathrm{P}=0.020)$ and advanced $\mathrm{pT}$ stage $(\mathrm{P}<0.001)$ were significantly associated with poorer prognosis (Table III). In multivariate analysis of the prognostic factors, strong CDCA1 expression $(\mathrm{P}=0.008)$ and higher $\mathrm{pT}$ stage $(\mathrm{P}<0.001)$ were identified as independent prognostic factors (Table III).

Growth-inhibitory effects of siRNAs against CDCA1. To assess the role of CDCA1 in CRC cell growth and/or survival, we knocked down CDCA1 expression in the CDCA1-positive CRC cell lines SW480 and SW948 using two siRNAs against CDCA1 (si-CDCA1-1 and -2), along with two control siRNAs (siRNAs for EGFP and LUC). Transfection of si-CDCA1s into CRC cells reduced CDCA1 protein levels, and significantly reduced cell viability (Fig. 4). These results indicate that CDCA1 is indispensable for growth and survival of CRC cells.

To further investigate the mechanisms of CRC cell growth suppression caused by reduced CDCA1 expression, we measured the DNA contents using flow cytometric analysis after CDCA1 (si-CDCA1-1) siRNA transfection into CRC cells. We observed 
Table III. Cox's proportional hazards model analysis of prognostic factors in patient with colorectal cancers.

\begin{tabular}{|c|c|c|c|}
\hline Variables & Hazards ratio $(95 \% \mathrm{CI})$ & Unfavorable/favorable & P-value \\
\hline \multicolumn{4}{|c|}{ Univariate analysis } \\
\hline CDCA1 & $1.813 \quad(1.097-2.997)$ & Strong/weak, absent & $0.020^{\mathrm{a}}$ \\
\hline Gender & $0.775 \quad(0.464-1.295)$ & Male/female & 0.330 \\
\hline Age (years) & $1.189(0.725-1.952)$ & $\geq 65 /<65$ & 0.493 \\
\hline Histologic type & $0.643 \quad(0.258-1.604)$ & tub1, tub2, pap/por1, por2, sig, others & 0.344 \\
\hline pT factor & $14.222(3.477-58.166)$ & $\mathrm{T} 3+\mathrm{T} 4 / \mathrm{Tis}+\mathrm{T} 1+\mathrm{T} 2$ & $<0.001^{\mathrm{a}}$ \\
\hline $\mathrm{pN}$ factor & $1.299 \quad(0.706-2.393)$ & $\mathrm{N} 2 / \mathrm{N} 0+\mathrm{N} 1$ & 0.401 \\
\hline \multicolumn{4}{|c|}{ Multivariate analysis } \\
\hline CDCA1 & $1.977(1.195-3.269)$ & Strong/weak, absent & $0.008^{\mathrm{a}}$ \\
\hline pT factor & $14.877(3.636-60.874)$ & $\mathrm{T} 3+\mathrm{T} 4 / \mathrm{Tis}+\mathrm{T} 1+\mathrm{T} 2$ & $<0.001^{\mathrm{a}}$ \\
\hline
\end{tabular}

${ }^{\mathrm{a}} \mathrm{P}<0.05$ (Fisher's exact test). tub, tubular adenocarcinoma; tub1, well-differentiated type; tub2, moderately-differentiated type; pap, papillary adenocarcinoma; por, poorly-differentiated adenocarcinoma; por1, solid type; por2, non-solid type; sig, signet ring cell carcinoma.
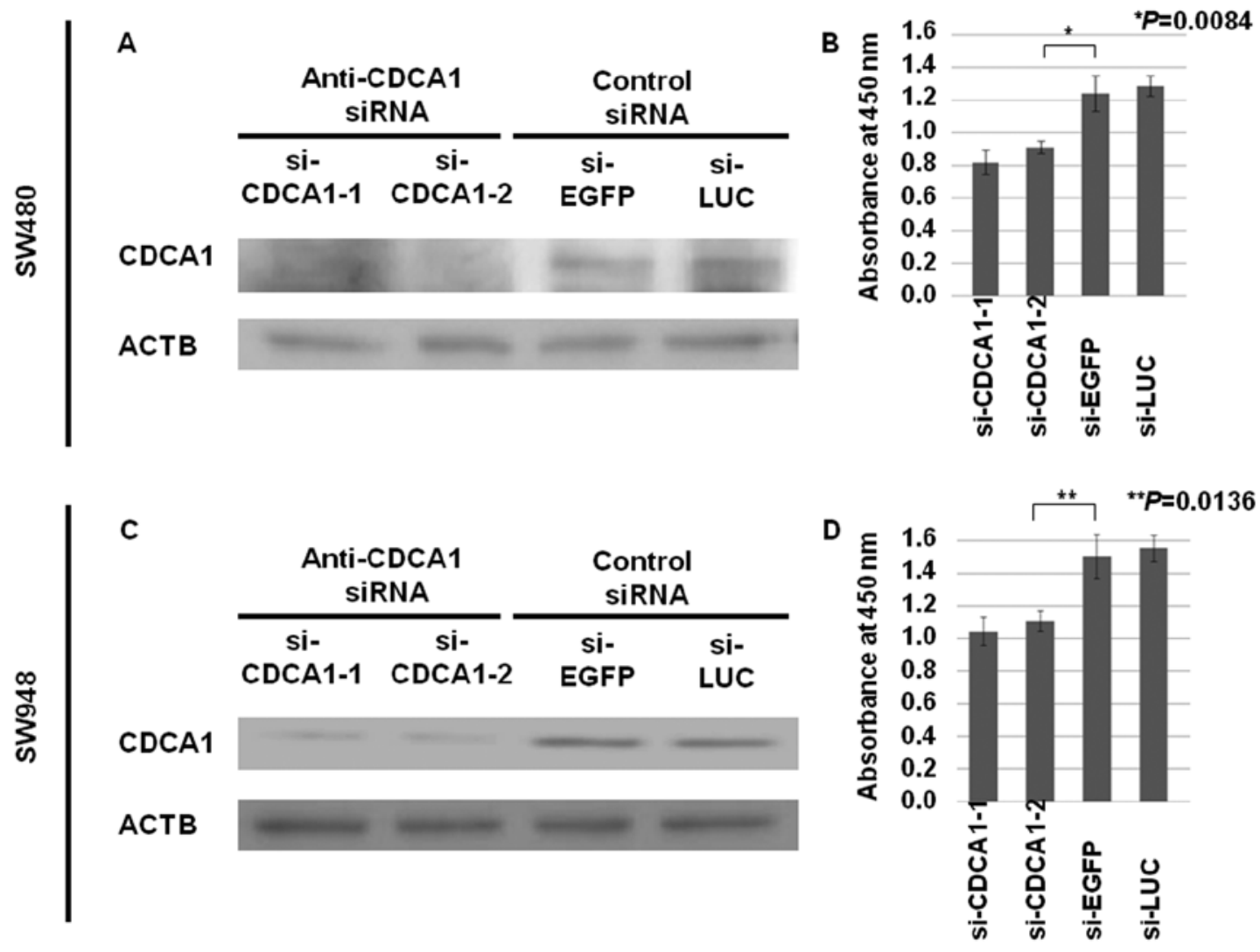

Figure 4. Growth-inhibitory effects of CDCA1 siRNAs on CRC cells. Western blotting showing suppression of CDCA1 expression mediated by CDCA1specific siRNAs, si-CDCA1-1 and si-CDCA1-2, in CRC cell lines SW480 and SW948, respectively. ACTB protein expression served as a quantity control for protein levels (A and C). MTT assay demonstrating a decrease in cell numbers by knockdown of CDCA1 in SW480 cells (si-CDCA1-2 and si-EGFP; P=0.0084; unpaired t-test) and SW948 cells (si-CDCA1-2 and si-EGFP; $\mathrm{P}=0.0136$; unpaired t-test) (B and D).

an increased sub-G1 fraction in SW480 and SW948 cells transfected with si-CDCA1-1, compared with those transfected with control siRNAs (Fig. 5A). In addition, caspase 3/7 assay detected an increase in caspase 3/7 activity, whereas TUNEL assay showed an increase in TUNEL staining in SW480 and
SW948 cells transfected with siRNAs for CDCA1, compared with those transfected with control siRNAs. These results independently demonstrated activation of caspase cascades and subsequent DNA fragmentation in CRC cells transfected with siRNAs for CDCA1 (Fig. 5B and C). 
SW480

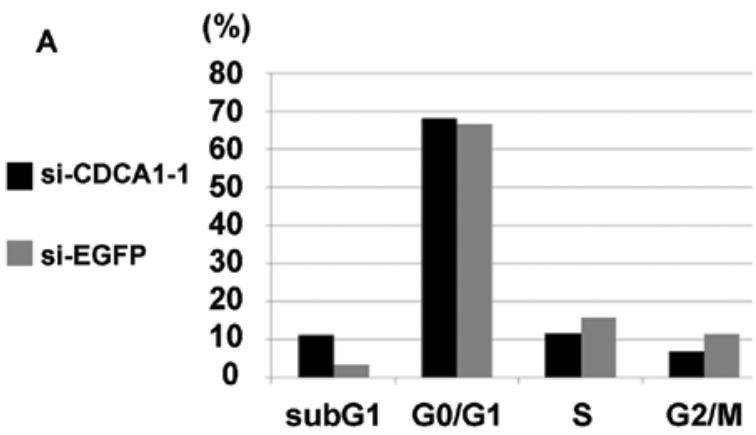

B

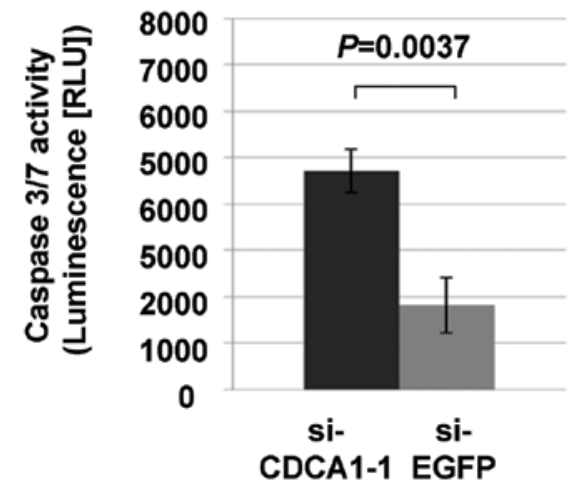

C

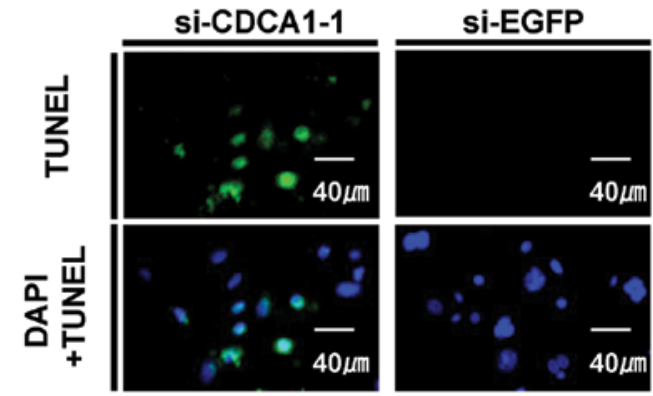

(\%)

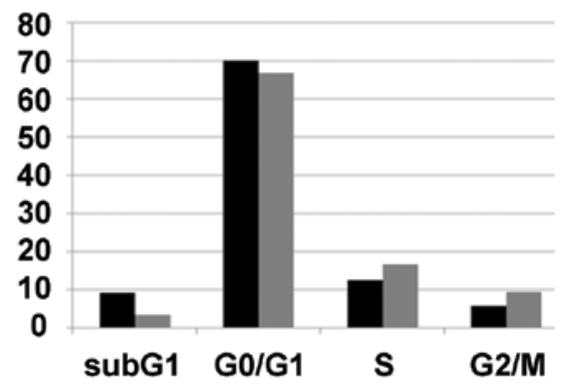

Figure 5. CDCA1 knockdown-induced apoptosis of CRC cells. (A) DNA content of SW480 and SW948 cells after CDCA1-siRNA transfection. (B) Caspase 3/7 activity of SW480 and SW948 cells after CDCA1-siRNA transfection. (C) TUNEL assay of SW480 and SW948 cells after CDCA1-siRNA transfection.

\section{Discussion}

Significant advances in the development of molecular-targeting drugs for cancer therapy have been achieved in the last two decades. However, the number of patients that respond to the presently available treatments is limited, and a subset of the patients suffers from severe adverse reactions without clinical benefits. Therefore, it is critical to develop new anticancer agents that are highly specific to malignant cells and have a minimum risk of adverse side effects.

We previously reported that CDCA1 overexpression plays a key role in the proliferation of lung cancer. CDCA1 is one of the highly conserved components of nuclear division cycle complex and is categorized as an oncoantigen that can induce peptide-specific CTLs against solid tumors $(16,39)$. In the present study, we demonstrated that CDCA1 expression is also elevated in many CRC tissues. Similar to previous findings in lung cancer cells, knocking down CDCA1 with siRNA inhibited CRC cell growth, which suggests increased CDCA1 expression is necessary for CRC cell proliferation and/or survival. CDCA1 protein functions at kinetochores for stable microtubule attachment and stable kinetochore localization of centromere-associated protein E (CENP-E) in the cervical cancer cell line HeLa (45-47). When CDCA1 expression was reduced in these cells, kinetochores failed to form attachments with spindle microtubules, which were followed by aberrant chromosome segregation, a prolonged mitotic blockade, and cell death (45-47). This aberrant exit from mitosis has characteristics of both apoptosis and catastrophe (45). Recent investigations also demonstrated that knocking down centrosomal proteins such as aurora A and ninein in HeLa cells led to aberrant spindle formation and subsequent cell death, 
which are accompanied by several features of apoptosis (48). Therefore, we presume that apoptosis of CRC cells induced by CDCA1 expression inhibition by siRNA could result from a similar mechanism. However, further studies are needed to clarify the relationship between CDCA1 suppression and mitotic catastrophe or apoptosis. Many proteins that regulate mitosis are aberrantly expressed in human tumor cells when compared with their normal counterparts, and some of these function as oncogenes, such as aurora kinase and polo-like kinase $(49,50)$. Some of these proteins are potential targets for anticancer agents. For example, highly conserved aurora kinases are critical mitotic regulators (49). Several aurora kinase inhibitors, including ZM447439, Hesperadin and VX-680 have been described as anticancer drugs (49). CDCA1 could serve as a valuable target for molecular-targeted therapies, as well as peptide vaccine immunotherapy for CRC.

In addition, we demonstrated that CDCA1 was highly expressed in $55.3 \%$ of surgically resected samples obtained from CRC patients and this overexpression was associated with a poorer prognosis. A publicly available microarray database (http://www.prognoscan.org/) revealed a significant correlation between high $C D C A 1$ expression and a reduced OS period for CRC patients (dataset no. GSE17536; $\mathrm{P}=0.028054$ ), which independently supports our data that CDCA1 expression has prognostic value for CRC patients. CDCA1 positivity in CRC tissues could provide a clinical prognostic indicator that warrants intensive follow-up in patients and/or addition of adjuvant chemotherapy after surgical treatments.

To examine the mechanisms of CDCA1 activation and overexpression in CRCs, we searched previous publications and databases for $C D C A 1$ including the $\mathrm{CGH}$ and genome sequencing (http://cancer.sanger.ac.uk/cosmic/gene/) databases. Missense mutation was indicated in 6 of the $652 \mathrm{CRCs}$ (0.92\%), but no CDCA1 gene amplification or translocation was reported in CRCs. Therefore, we speculate that overexpression of CDCA1 may be primarily caused by epigenetic mechanism. Further analysis of CDCA1, including screening using functional assays for an activating mutation or epigenetic regulating mechanisms of CDCA1 may further clarify the oncogenic function of CDCA1.

In conclusion, our data suggest that CDCA1 contributes to the viability and malignant potential of CRC cells, and is a clinically promising prognostic biomarker in addition to a potential molecular target for treating CRC.

\section{References}

1. Jemal A, Bray F, Center MM, Ferlay J, Ward E and Forman D: Global cancer statistics. CA Cancer J Clin 61: 69-90, 2011.

2. Van Cutsem E, Köhne CH, Hitre E, Zaluski J, Chang Chien CR, Makhson A, D'Haens G, Pintér T, Lim R, Bodoky G, Roh JK, Folprecht G, Ruff P, Stroh C, Tejpar S, Schlichting M, Nippgen J and Rougier P: Cetuximab and chemotherapy as initial treatment for metastatic colorectal cancer. N Engl J Med 360: 1408-1417, 2009.

3. Bokemeyer C, Van Cutsem E, Rougier P, Ciardiello F, Heeger S, Schlichting M, Celik I and Köhne CH: Addition of cetuximab to chemotherapy as first-line treatment for KRAS wild-type metastatic colorectal cancer: pooled analysis of the CRYSTAL and OPUS randomised clinical trials. Eur J Cancer 48: 1466-1475, 2012.

4. Daigo Y and Nakamura Y: From cancer genomics to thoracic oncology: discovery of new biomarkers and therapeutic targets for lung and esophageal carcinoma. Gen Thorac Cardiovasc Surg 56: 43-53, 2008.
5. Daigo Y, Takano A, Teramoto K, Chung S and Nakamura Y: A systematic approach to the development of novel therapeutics for lung cancer using genomic analyses. Clin Pharmacol Ther 94: 218-223, 2013.

6. Kikuchi T, Daigo Y, Katagiri T, et al: Expression profiles of non-small cell lung cancers on cDNA microarrays: identification of genes for prediction of lymph-node metastasis and sensitivity to anti-cancer drugs. Oncogene 22: 2192-2205, 2003.

7. Kakiuchi S, Daigo Y, Ishikawa N, et al: Prediction of sensitivity of advanced non-small cell lung cancers to gefitinib (Iressa, ZD1839). Hum Mol Genet 13: 3029-3043, 2004.

8. Kikuchi T, Daigo Y, Ishikawa N, et al: Expression profiles of metastatic brain tumor from lung adenocarcinomas on cDNA microarray. Int J Oncol 28: 799-805, 2006.

9. Taniwaki M, Daigo Y, Ishikawa N, et al: Gene expression profiles of small-cell lung cancers: molecular signatures of lung cancer. Int J Oncol 29: 567-575, 2006.

10. Yamabuki T, Daigo Y, Kato T, et al: Genome-wide gene expression profile analysis of esophageal squamous cell carcinomas. Int J Oncol 28: 1375-1384, 2006.

11. Suzuki C, Daigo Y, Kikuchi T, Katagiri T and Nakamura Y: Identification of COX17 as a therapeutic target for non-small cell lung cancer. Cancer Res 63: 7038-7041, 2003.

12. Kato T, Daigo Y, Hayama S, et al: A novel human tRNAdihydrouridine synthase involved in pulmonary carcinogenesis. Cancer Res 65: 5638-5646, 2005.

13. Furukawa C, Daigo Y, Ishikawa N, et al: Plakophilin 3 oncogene as prognostic marker and therapeutic target for lung cancer. Cancer Res 65: 7102-7110, 2005.

14. Suzuki C, Daigo Y, Ishikawa N, et al: ANLN plays a critical role in human lung carcinogenesis through the activation of RHOA and by involvement in the phosphoinositide 3-kinase/AKT pathway. Cancer Res 65: 11314-11325, 2005.

15. Takahashi K, Furukawa C, Takano A, et al: The neuromedin $\mathrm{U}$-growth hormone secretagogue receptor $1 \mathrm{~b} /$ neurotensin receptor 1 oncogenic signaling pathway as a therapeutic target for lung cancer. Cancer Res 66: 9408-9419, 2006.

16. Hayama S, Daigo Y, Kato T, et al: Activation of CDCA1-KNTC2, members of centromere protein complex, involved in pulmonary carcinogenesis. Cancer Res 66: 10339-10348, 2006.

17. Kato T, Hayama S, Yamabuki T, et al: Increased expression of IGF-II mRNA-binding protein 1 is associated with the tumor progression in patients with lung cancer. Clin Cancer Res 13: 434-442, 2007.

18. Hayama S, Daigo Y, Yamabuki T, et al: Phosphorylation and activation of cell division cycle associated 8 by aurora kinase B plays a significant role in human lung carcinogenesis. Cancer Res 67: 4113-4122, 2007

19. Taniwaki M, Takano A, Ishikawa N, et al: Activation of KIF4A as a prognostic biomarker and therapeutic target for lung cancer. Clin Cancer Res 13: 6624-6631, 2007.

20. Kato T, Sato N, Hayama S, et al: Activation of holliday junction recognizing protein involved in the chromosomal stability and immortality of cancer cells. Cancer Res 67: 8544-8553, 2007.

21. Kato T, Sato N, Takano A, et al: Activation of placenta-specific transcription factor distal-less homeobox 5 predicts clinical outcome in primary lung cancer patients. Clin Cancer Res 14: 2363-2370, 2008.

22. Dunleavy EM, Roche D, Tagami H, et al: HJURP is a cell-cycledependent maintenance and deposition factor of CENP-A at centromeres. Cell 137: 485-497, 2009.

23. Hirata D, Yamabuki T, Miki D, et al: Involvement of epithelial cell transforming sequence 2 (ECT2) oncoantigen in lung and esophageal cancer progression. Clin Cancer Res 15: 256-266, 2009.

24. Sato N, Koinuma J, Fujita M, et al: Activation of WD repeat and high-mobility group box DNA binding protein 1 in pulmonary and esophageal carcinogenesis. Clin Cancer Res 16: 226-239, 2010.

25. Nguyen MH, Koinuma J, Ueda K, et al: Phosphorylation and activation of cell division cycle associated 5 by mitogen-activated protein kinase play a crucial role in human lung carcinogenesis. Cancer Res 70: 5337-5347, 2010.

26. Aragaki M, Takahashi K, Akiyama H, et al: Characterization of a cleavage stimulation factor, 3' pre-RNA, subunit 2, $64 \mathrm{kDa}$ (CSTF2) as a therapeutic target for lung cancer. Clin Cancer Res 7: 5889-5900, 2011.

27. Masuda K, Takano A, Oshita H, et al: Chondrolectin is a novel diagnostic biomarker and a therapeutic target for lung cancer. Clin Cancer Res 17: 7712-7722, 2011. 
28. Fujitomo T, Daigo Y, Matsuda K, Ueda K and Nakamura Y: Critical function for nuclear envelope protein TMEM209 in human pulmonary carcinogenesis. Cancer Res 72: 4110-4118, 2012.

29. Oshita H, Nishino R, Takano A, et al: RASEF is a novel diagnostic biomarker and a therapeutic target for lung cancer. Mol Cancer Res 11: 937-951, 2013.

30. Ishikawa N, Daigo Y, Yasui W, et al: ADAM8 as a novel serological and histochemical marker for lung cancer. Clin Cancer Res 10: 8363-8370, 2004.

31. Ishikawa N, Daigo Y, Takano A, et al: Increases of amphiregulin and transforming growth factor-alpha in serum as predictors of poor response to gefitinib among patients with advanced non-small cell lung cancers. Cancer Res 65: 9176-9184, 2005.

32. Yamabuki T, Takano A, Hayama S, et al: Dickkopf-1 as a novel serologic and prognostic biomarker for lung and esophageal carcinomas. Cancer Res 67: 2517-2525, 2007.

33. Ishikawa N, Takano A, Yasui W, et al: Cancer-testis antigen lymphocyte antigen 6 complex locus $\mathrm{K}$ is a serologic biomarker and a therapeutic target for lung and esophageal carcinomas. Cancer Res 67: 11601-11611, 2007.

34. Takano A, Ishikawa N, Nishino R, et al: Identification of nectin-4 oncoprotein as a diagnostic and therapeutic target for lung cancer. Cancer Res 69: 6694-6703, 2009.

35. Sato N, Yamabuki T, Takano A, et al: Wnt inhibitor Dickkopf-1 as a target for passive cancer immunotherapy. Cancer Res 70 . 5326-5336, 2010

36. Nishino $\mathrm{R}$, Takano A, Oshita $\mathrm{H}$, et al: Identification of Epstein-Barr virus-induced gene 3 as a novel serum and tissue biomarker and a therapeutic target for lung cancer. Clin Cancer Res 17: 6272-6286, 2011.

37. Suda T, Tsunoda T, Daigo Y, Nakamura Y and Tahara H: Identification of human leukocyte antigen-A24-restricted epitope peptides derived from gene products upregulated in lung and esophageal cancers as novel targets for immunotherapy. Cancer Sci 98: 1803-1808, 2007.

38. Mizukami Y, Kono K, Daigo Y, et al: Detection of novel cancertestis antigen-specific T-cell responses in TIL, regional lymph nodes, and PBL in patients with esophageal squamous cell carcinoma. Cancer Sci 99: 1448-1454, 2008.

39. Harao M, Hirata S, Irie A, et al: HLA-A2-restricted CTL epitopes of a novel lung cancer-associated cancer testis antigen, cell division cycle associated 1 , can induce tumor-reactive CTL. Int J Cancer 123: 2616-2625, 2008.
40. Kono K, Mizukami Y, Daigo Y, et al: Vaccination with multiple peptides derived from novel cancer-testis antigens can induce specific T-cell responses and clinical responses in advanced esophageal cancer. Cancer Sci 100: 1502-1509, 2009.

41. Yokomine K, Senju S, Nakatsura T, et al: The forkhead box M1 transcription factor, as a possible immunotherapeutic tumorassociated antigen. Int J Cancer 126: 2153-2163, 2010.

42. Tomita Y,Imai K, Senju S, et al: A novel tumor-associated antigen, cell division cycle 45-like can induce cytotoxic T lymphocytes reactive to tumor cells. Cancer Sci 102: 697-705, 2011.

43. Kaneko N, Miura K, Gu Z, Karasawa H, Ohnuma S, Sasaki H, Tsukamoto N, Yokoyama S, Yamamura A, Nagase H, Shibata C, Sasaki I and Horii A: siRNA-mediated knockdown against CDCA1 and KNTC2, both frequently overexpressed in colorectal and gastric cancers, suppresses cell proliferation and induces apoptosis. Biochem Biophys Res Commun 390: 1235-1240, 2009.

44. van Duin M, Broyl A, de Knegt Y, Goldschmidt H, Richardson PG, Hop WC, van der Holt B, Joseph-Pietras D, Mulligan G, Neuwirth R, Sahota SS and Sonneveld P: Cancer testis antigens in newly diagnosed and relapse multiple myeloma: prognostic markers and potential targets for immunotherapy. Haematologica 96: 1662-1669, 2011.

45. DeLuca JG, Moree B, Hickey JM, Kilmartin JV and Salmon ED hNuf2 inhibition blocks stable kinetochore-microtubule attachment and induces mitotic cell death in HeLa cells. J Cell Biol 159: 549-555, 2002

46. DeLuca JG, Howell BJ, Canman JC, Hickey JM, Fang G and Salmon ED: Nuf2 and $\mathrm{Hecl}$ are required for retention of the checkpoint proteins Mad1 and Mad2 to kinetochores. Curr Biol 13: 2103-2109, 2003.

47. Liu D, Ding X, Du J, Cai X, Huang Y, Ward T, Shaw A, Yang Y, Hu R, Jin C and Yao X: Human NUF2 interacts with centromere-associated protein $\mathrm{E}$ and is essential for a stable spindle microtubule-kinetochore attachment. J Biol Chem 282: 21415-21424, 2007.

48. Kimura M, Yoshioka T, Saio M, Banno Y, Nagaoka H and Okano Y: Mitotic catastrophe and cell death induced by depletion of centrosomal proteins. Cell Death Dis 4: e603, 2013.

49. Keen $\mathrm{N}$ and Taylor S: Aurora-kinase inhibitors as anticancer agents. Nat Rev Cancer 4: 927-936, 2004.

50. Jang YJ, Kim YS and Kim WH: Oncogenic effect of Polo-like kinase 1 expression in human gastric carcinomas. Int J Oncol 29: 589-594, 2006 